\title{
Chaotic Motion in Forced Duffing System Subject to Linear and Nonlinear Damping
}

\author{
Tai-Ping Chang \\ Department of Construction Engineering, National Kaohsiung First University of Science and Technology, Kaohsiung, Taiwan \\ Correspondence should be addressed to Tai-Ping Chang; tpchang@ccms.nkfust.edu.tw
}

Received 6 December 2016; Accepted 16 January 2017; Published 31 January 2017

Academic Editor: Jonathan N. Blakely

Copyright (C) 2017 Tai-Ping Chang. This is an open access article distributed under the Creative Commons Attribution License, which permits unrestricted use, distribution, and reproduction in any medium, provided the original work is properly cited.

\begin{abstract}
This paper investigates the chaotic motion in forced Duffing oscillator due to linear and nonlinear damping by using Melnikov technique. In particular, the critical value of the forcing amplitude of the nonlinear system is calculated by Melnikov technique. Further, the top Lyapunov exponent of the nonlinear system is evaluated by Wolf's algorithm to determine whether the chaotic phenomenon of the nonlinear system actually occurs. It is concluded that the chaotic motion of the nonlinear system occurs when the forcing amplitude exceeds the critical value, and the linear and nonlinear damping can generate pronounced effects on the chaotic behavior of the forced Duffing oscillator.
\end{abstract}

\section{Introduction}

The study of chaotic motion in nonlinear systems has been a popular area of research during the last few decades. Many investigations have been performed on different nonlinear chaotic systems to understand the complex behavior of these systems. Three of the fundamental forced oscillators, Duffing, Van der Pol, and Rayleigh oscillators, have been extensively examined since lots of dynamic characteristics embedded in the physical systems can be realized from these three systems [1-3]. Among them, forced Duffing oscillator is the most useful nonlinear dynamical systems, which is considered as a prototype model for various physical and engineering problems such as dynamics of a buckled elastic beam, particle in a forced double well, particle in a plasma, and a defect in solids [4]. More recently, Wang et al. [57] show that many nonlinear dynamical problems can be reduced to Duffing system for structures subject to both free and forced excitation. Therefore, the Duffing oscillator is still an interesting model to study for discovering the characteristics of chaos in nonlinear physical systems despite lots of investigations have been performed on this model for so many years. The Duffing oscillator can be interpreted as a damped oscillator with a complicated potential. The damping or dissipation here is very important as it decides the border of stability and instability. In the past, most of the studies on Duffing system have been performed by considering the linear viscous damping. Several researches have been reported on the chaotic motion of forced Duffing equations [8-10]. However, the consideration of nonlinear damping is quite necessary in various engineering applications such as drag forces in flow induced vibrations [4] and vibration isolators [11]. Among others, some researches have made the contributions to the chaotic behavior of Duffing oscillator due to nonlinear damping [12-21].

Generally speaking, the Melnikov method is very useful for detecting the presence of transverse homoclinic orbits and the occurrence of homoclinic bifurcations. However, the traditional Melnikov methods strongly depend on the small perturbation parameters so that these methods are limited in coping with the systems with strong nonlinearities. Liu et al. [22] presents a procedure to investigate the chaos and subharmonic resonance of strongly nonlinear practical systems by using a homotopy method that is adopted to extend the Melnikov functions to the strongly nonlinear systems.

In the present study, the chaotic behavior of a forced Duffing oscillator subjected to linear and nonlinear damping is investigated by using Melnikov technique [23, 24]. In particular, the critical values of the forcing amplitude of the 
nonlinear system is calculated by Melnikov technique. The Lyapunov exponents of the nonlinear system are evaluated by Wolf's algorithm [25] to determine whether the chaotic phenomenon of the nonlinear system actually occurs.

Although the Melnikov method is used here only for Duffing system without the control action, however, it is known that control action is an important direction of the nonlinear dynamics field. Among others, recently Wang and Li [26] investigated the nonlinear dynamical characteristics of the Duffing-Van der Pol oscillator subject to both external and parametric excitations with time delayed feedback control by using the multiple scale method. Actually, the Melnikov method can also be used for Duffing system with the feedback control. Yagasaki [27] considered a pendulum subjected to linear feedback control with periodic desired motions. He studied local bifurcations of harmonics and subharmonics using the second-order averaging method and Melnikov's method.

\section{Governing Equation of Nonlinear System}

In many engineering and physics fields, there are lots of models can be converted into the following forced Duffing equation:

$$
\ddot{x}-\omega_{0}^{2} x+D \dot{x}+\gamma \dot{x}^{3}+\beta x^{3}=F \cos (\omega t),
$$

where $-\omega_{0}^{2}$ denotes the linear stiffness constant of the system, $D$ is linear damping coefficient, $\gamma$ is the nonlinear damping coefficient, $\beta$ is the nonlinear stiffness constant, and $F$ and $\omega$ are the external amplitude and driving frequency, respectively. In the present study, $D, \gamma$, and $F$ are assumed to be small parameters. Hence, a transformation $D \rightarrow \varepsilon D, \gamma \rightarrow$ $\varepsilon \gamma, F \rightarrow \varepsilon F$ is adopted in order to apply the first-order perturbation scheme of the Melnikov method. Therefore, (1) can be rewritten as

$$
\begin{aligned}
& \dot{x}=y, \\
& \dot{y}=\omega_{0}^{2} x-\beta x^{3}+\varepsilon\left(-D y-\gamma y^{3}+F \cos (\omega t)\right) .
\end{aligned}
$$

When $\varepsilon=0$, an unperturbed system can be obtained as follows:

$$
\begin{aligned}
& \dot{x}=y, \\
& \dot{y}=\omega_{0}^{2} x-\beta x^{3} .
\end{aligned}
$$

The system of (3) corresponds to a Hamiltonian system with a potential function

$$
V(x)=-\frac{\omega_{0}^{2}}{2} x^{2}+\frac{\beta}{4} x^{4} .
$$

The unperturbed system of (4) has three equilibrium points: one saddle $(0,0)$ and two centers $\left( \pm \omega_{0} / \sqrt{\beta}, 0\right)$.

\section{Chaotic Motion of Forced Duffing Oscillator}

By integrating system (3), the solution of unperturbed system (3) can be written as follows:

$$
\begin{aligned}
& x_{0}(t)=\sqrt{\frac{2}{\beta}} \omega_{0} \operatorname{sech}\left(\omega_{0} t\right), \\
& \dot{x}_{0}(t)=y_{0}(t)=\sqrt{\frac{2}{\beta}} \omega_{0}^{2} \operatorname{sech}\left(\omega_{0} t\right) \tanh \left(\omega_{0} t\right) .
\end{aligned}
$$

Now we can use Melnikov's method [23, 24] to investigate the homoclinic bifurcation in the forced Duffing oscillator system with linear and nonlinear damping as given in (1). The Melnikov function measures the distance between the stable and unstable manifolds in the Poincare section, and to preserve the homoclinic loops under a perturbation requires that, at $t_{0}$, if $M\left(t_{0}\right)$ has a simple zero, then a homoclinic bifurcation occurs, implying that the chaotic motion occurs. The Melnikov function for the forced Duffing oscillator system shown in (1) can be obtained as follows:

$$
\begin{aligned}
M\left(t_{0}\right)= & -D \int_{-\infty}^{\infty} y_{0}^{2} d t-\gamma \int_{-\infty}^{\infty} y_{0}^{4} d t \\
& +F \int_{-\infty}^{\infty} y_{0} \cos \left(\omega\left(t-t_{0}\right)\right) d t
\end{aligned}
$$

where $t_{0}$ is the cross-section time of the Poincare map and $t_{0}$ can be interpreted as the initial time of the forcing term. By substituting (5) into (6) and calculating the integral, we can get the Melnikov function as follows:

$$
M\left(t_{0}\right)=-D I_{1}-\gamma I_{2}+F I_{3} \sin \left(\omega t_{0}\right),
$$

where

$$
\begin{aligned}
& I_{1}=\frac{2 \omega_{0}^{4}}{\beta} \int_{-\infty}^{\infty} \operatorname{sech}^{2}\left(\omega_{0} t\right) \tanh ^{2}\left(\omega_{0} t\right) d t=\frac{4 \omega_{0}^{3}}{3 \beta}, \\
& I_{2}=\frac{4 \omega_{0}^{8}}{\beta^{2}} \int_{-\infty}^{\infty} \operatorname{sech}^{4}\left(\omega_{0} t\right) \tanh ^{4}\left(\omega_{0} t\right) d t=\frac{16 \omega_{0}^{7}}{35 \beta^{2}}, \\
& I_{3}=-\sqrt{\frac{2}{\beta}} \omega_{0} \int_{-\infty}^{\infty} \operatorname{sech}\left(\omega_{0} t\right) \tanh \left(\omega_{0} t\right) \sin (\omega t) d t \\
& =-\frac{\pi \omega \sqrt{2 \beta}}{\beta} \operatorname{sech}\left(\frac{\pi \omega}{2 \omega_{0}}\right) \text {. }
\end{aligned}
$$

The simple zeros of (7) give the critical value of the forcing amplitude $\left(F_{c}\right)$, and the first homoclinic bifurcation occurs when we cross the critical value by increasing the forcing amplitude, implying that the chaotic motion occurs in the system.

In (7), $M\left(t_{0}\right)$ will vanish if a solution can be detected for $t_{0}$, and $\left|\sin \left(\omega_{0} t\right)\right| \leq 1$; therefore, the critical value of the forcing amplitude $\left(F_{c}\right)$ can be obtained as follows:

$$
F=F_{c}=\left|\frac{D I_{1}+\gamma I_{2}}{I_{3}}\right|,
$$

where $I_{1}, I_{2}, I_{3}$ are given in (8). 
The main purpose of the present study is to investigate the chaotic motion of the nonlinear differential equation shown in (1) by evaluating the critical value of the forcing amplitude given in (9). The top Lyapunov exponent of the nonlinear system is evaluated by Wolf's algorithm [25] to check whether the chaotic phenomenon of the nonlinear system occurs. It is noticed that when the top Lyapunov exponent changes from negative to positive, the chaotic motion of the nonlinear system happens.

\section{Numerical Examples and Discussions}

First of all, the numerical values of the parameters in (1) are fixed as follows:

$$
\begin{aligned}
\omega_{0} & =1, \\
\beta & =1, \\
\gamma & =0, \\
\omega & =1 .
\end{aligned}
$$
follows:

In addition, the initial conditions in (1) are considered as

$$
\begin{aligned}
& x(0)=1.0, \\
& \dot{x}(0)=0.0 .
\end{aligned}
$$

Based on (9), the variations of critical values of forcing amplitude $\left(F_{c}\right)$ versus linear damping coefficient $D$, driving frequency $\omega$, and nonlinear coefficient $\beta$ are depicted in Figures 1(a)-1(c). As can be seen from Figure 1(a), $F_{c}$ increases linearly with the linear damping coefficient; however, $F_{c}$ varies nonlinearly with respect to the driving frequency as detected from Figure 1(b); first it decreases with the increase in $\omega$ and after reaching a minimum, it begins increasing. In Figure $1(\mathrm{c})$, the critical values of forcing amplitude $\left(F_{c}\right)$ decreases nonlinearly with respect to the nonlinear coefficient $\beta$. Now only the linear damping effect of the system is considered, and the parameters are fixed as follows: $\omega_{0}=$ $1, \beta=1, D=0.4, \gamma=0, \omega=1$. As can be seen from Figures 1(a)-1(c), the critical values of forcing amplitude $\left(F_{c}\right)$ are detected as 0.3012 ; hence, 0.305 is used for the numerical computations shown in Figures 3(a)-3(d) and 4(a) $-4(\mathrm{~d})$.

Now we include the nonlinear damping effect, that is, $\gamma=0.1$; while the other parameters remain the same as before, as can be seen in Figures 2(a)-2(c), the variations of critical values of forcing amplitude $\left(F_{c}\right)$ versus linear damping coefficient $D$, driving frequency $\omega$, and nonlinear coefficient $\beta$ are presented. It is noticed that the similar phenomena can be found by including the nonlinear damping effect except that the critical values of forcing amplitude $\left(F_{c}\right)$ are larger, which is quite reasonable.

In Figures 3(a)-3(d), we plot the top Lyapunov exponents, the time history, the phase portrait, and Poincare map of nonlinear system. As can be detected from Figure 3(a), the top Lyapunov exponent is computed as $\lambda_{1}=0.0088301$ which is positive, and the chaotic motion of the system will occur as shown in Figure 3(d). However, if the nonlinear

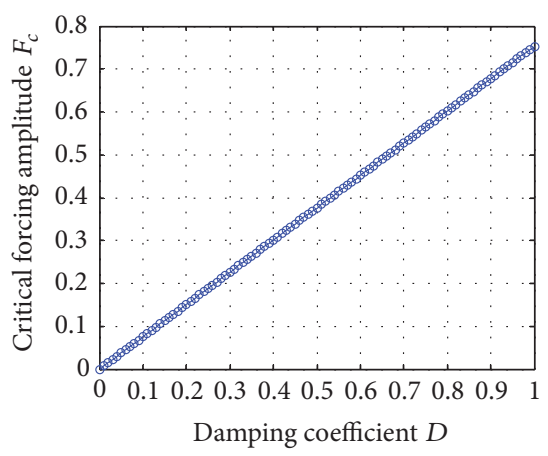

(a)

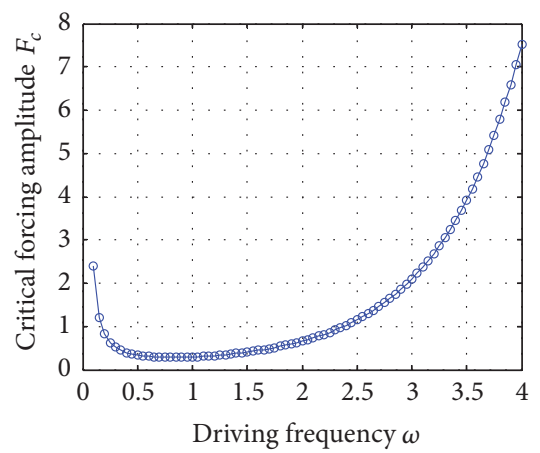

(b)

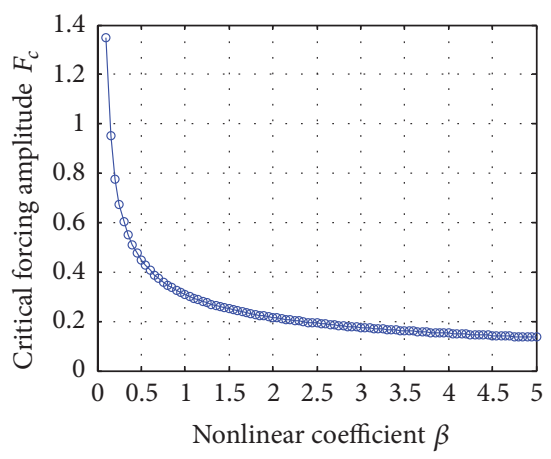

(c)

Figure 1: (a) Critical value of forcing amplitude $F_{c}$ versus linear damping coefficient $\left(\omega_{0}=1, \beta=1, \gamma=0, \omega=1\right)$. (b) Critical value of forcing amplitude $F_{c}$ versus driving frequency $\left(\omega_{0}=1, \beta=\right.$ $1, D=0.4, \gamma=0$ ). (c) Critical value of forcing amplitude $F_{c}$ versus nonlinear coefficient $\left(\omega_{0}=1, D=0.4, \gamma=0, \omega=1\right)$.

damping effect is considered, namely, $\gamma=0.1$, the other parameters remain the same; the top Lyapunov exponents, the time history, the phase portrait, and Poincare map of nonlinear system are presented in Figures 4(a)-4(d). As can be seen from Figure 4(a), the top Lyapunov exponent turns out to be $\lambda_{1}=-0.052549$, which is negative, and the periodic motion of the system can be detected as presented in Figure 4(d). Now let us use the numerical results shown in Figures 2(a)-2(c); the critical value of forcing amplitude $\left(F_{c}\right)$ of the nonlinear system with both linear and nonlinear damping is found as 0.3270 ; hence, 0.400 is adopted for the numerical computations shown in Figures 5(a)-5(d). 


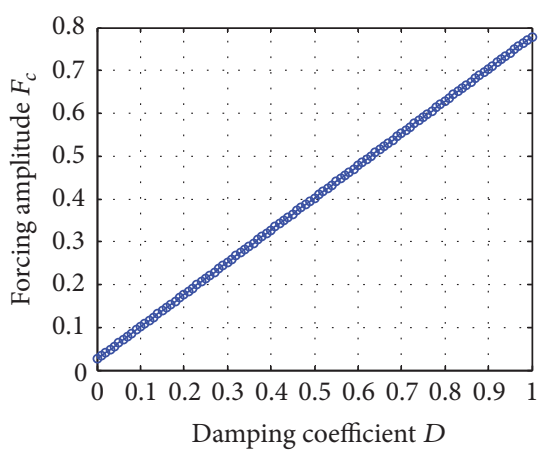

(a)

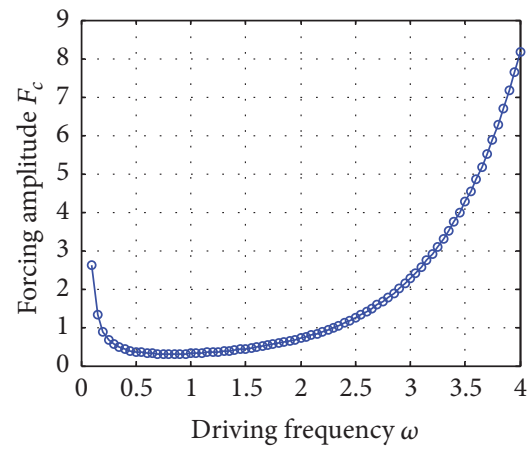

(b)

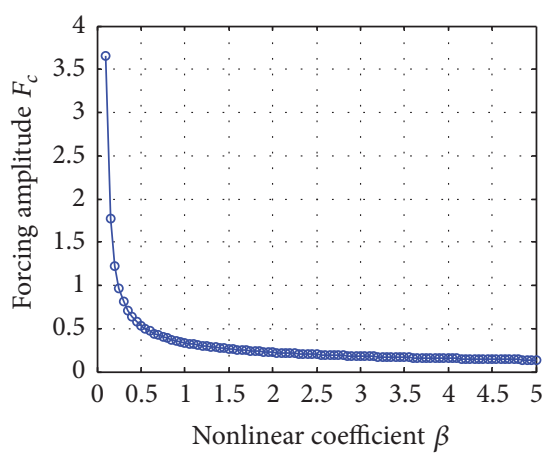

(c)

Figure 2: (a) Critical value of forcing amplitude $F_{c}$ versus linear damping coefficient $D\left(\omega_{0}=1, \beta=1, \gamma=0.1, \omega=1\right)$. (b) Critical value of forcing amplitude $F_{c}$ versus driving frequency $\left(\omega_{0}=1\right.$, $\beta=1, D=0.4, \gamma=0.1$ ). (c) Critical value of forcing amplitude $F_{c}$ versus nonlinear coefficient $\beta\left(\omega_{0}=1, D=0.4, \gamma=0.1, \omega=1\right)$.

In Figures 5(a)-5(d), the top Lyapunov exponents, the time history, the phase portrait, and Poincare map of nonlinear system are depicted under forcing amplitude $F=0.400$. The top Lyapunov exponent now changes from negative to positive as detected from Figure 5(a); as a result, the chaotic motion of the nonlinear system occurs again as shown in Figure 5(d), which is not surprising.

Now we switch the driving frequency of the system from $\omega=1.0$ to $\omega=2.0$ and consider first the linear damping effect only; in this case the following parameters are adopted: $\omega_{0}=1, \beta=1, D=0.4, \gamma=0, \omega=2$. As can be seen from Figure 1(b), the critical values of forcing amplitude $\left(F_{c}\right)$ is found as 0.6599 ; hence, 0.800 is used for the numerical

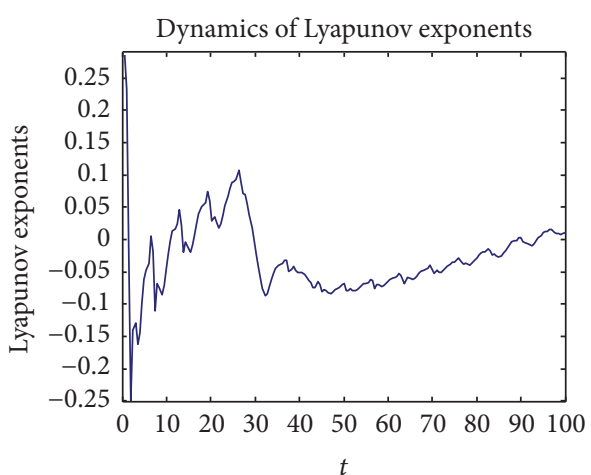

$\lambda_{1}=0.0088301$

(a)

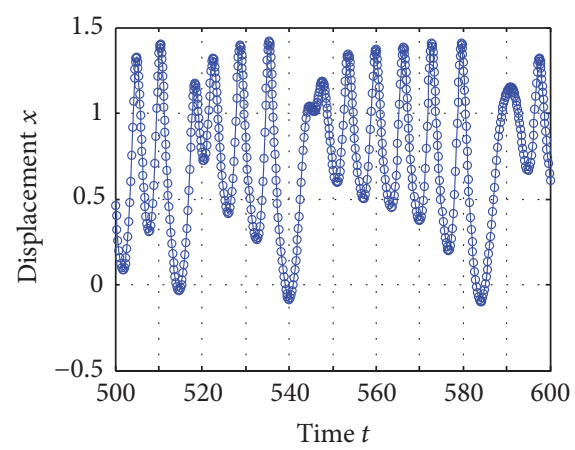

(b)

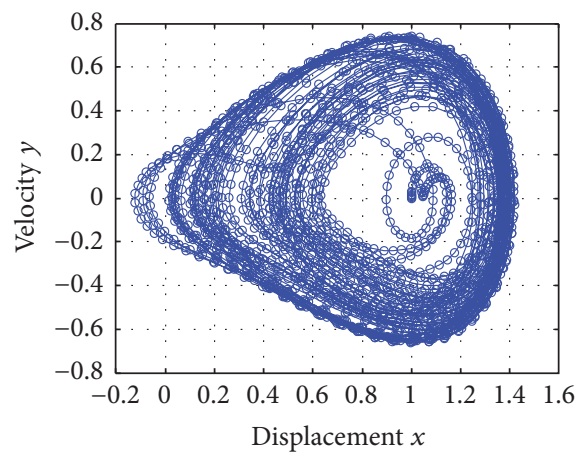

(c)

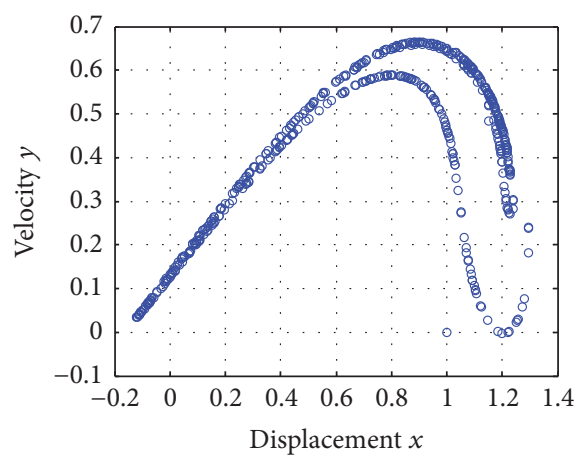

(d)

Figure 3: (a) Top Lyapunov exponents versus time $\left(\omega_{0}=1, \beta=1\right.$, $D=0.4, \gamma=0, \omega=1, F=0.305$ ). (b) Time history of nonlinear system $\left(\omega_{0}=1, \beta=1, D=0.4, \gamma=0, \omega=1, F=0.305\right)$. (c) Phase portrait of nonlinear system $\left(\omega_{0}=1, \beta=1, D=0.4, \gamma=0, \omega=1\right.$, $F=0.305)$. (d) Poincare map of nonlinear system $\left(\omega_{0}=1, \beta=1\right.$, $D=0.4, \gamma=0, \omega=1, F=0.305)$. 


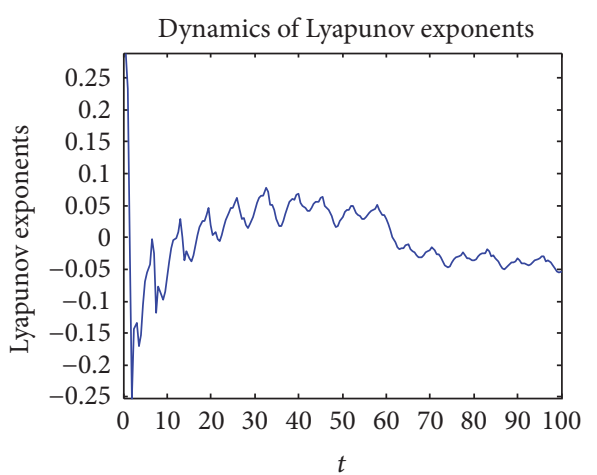

$-\lambda_{1}=-0.052549$

(a)

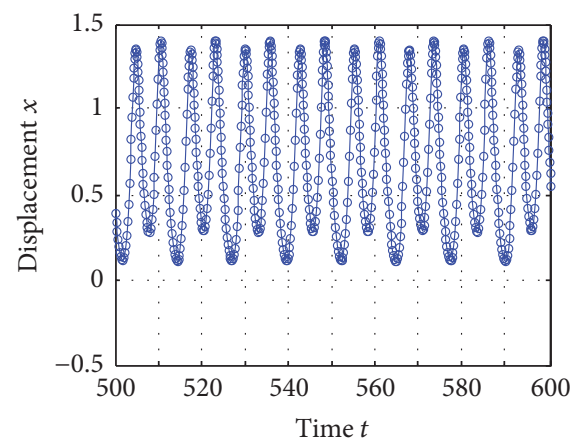

(b)

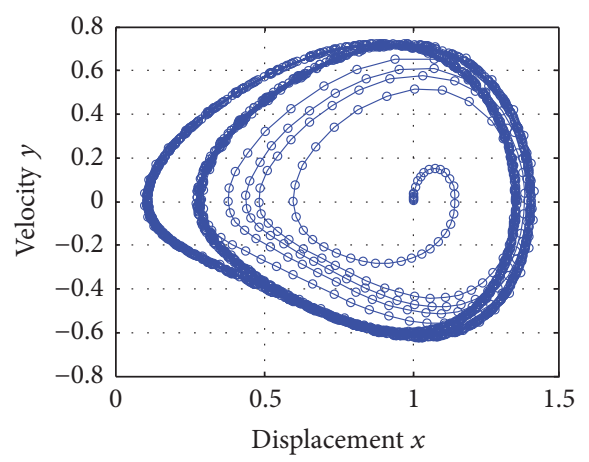

(c)

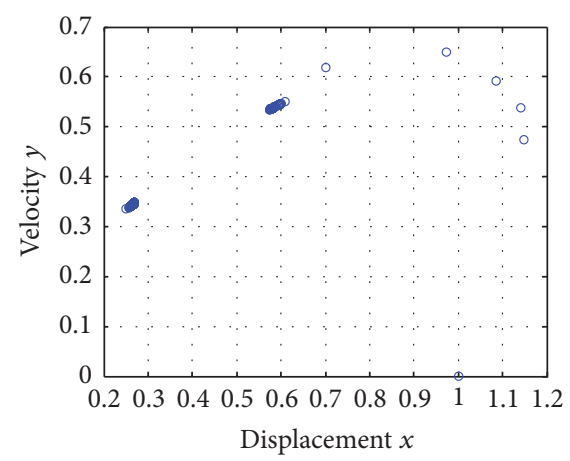

(d)

Figure 4: (a) Top Lyapunov exponents versus time $\left(\omega_{0}=1, \beta=1\right.$, $D=0.4, \gamma=0.1, \omega=1, F=0.305$ ). (b) Time history of nonlinear system $\left(\omega_{0}=1, \beta=1, D=0.4, \gamma=0.1, \omega=1, F=0.305\right)$. (c) Phase portrait of nonlinear system $\left(\omega_{0}=1, \beta=1, D=0.4, \gamma=0.1, \omega=1\right.$, $F=0.305)$. (d) Poincare map of nonlinear system $\left(\omega_{0}=1, \beta=1\right.$, $D=0.4, \gamma=0.1, \omega=1, F=0.305)$.

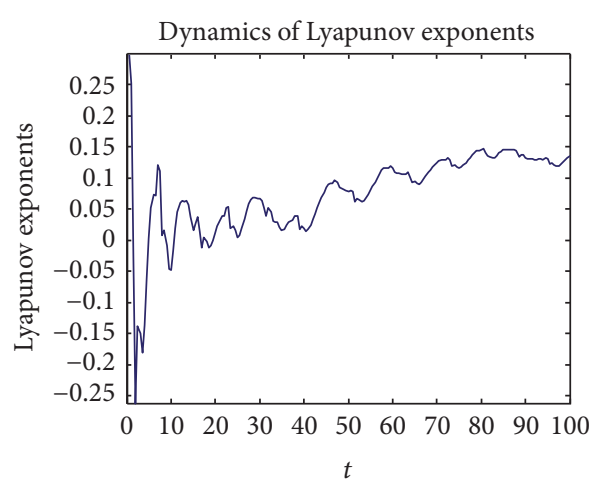

(a)

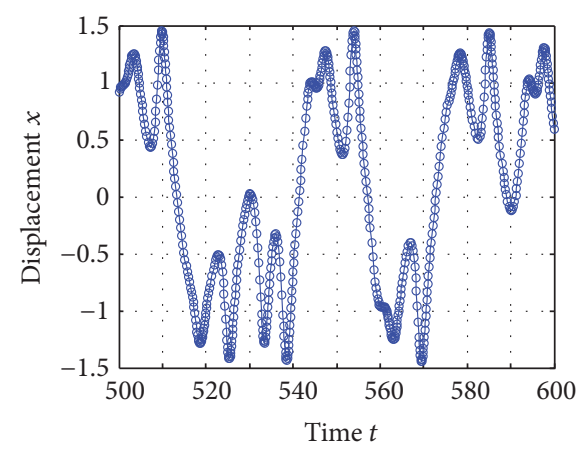

(b)

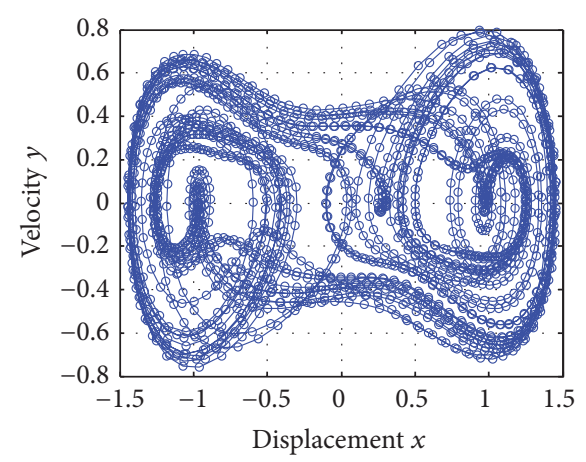

(c)

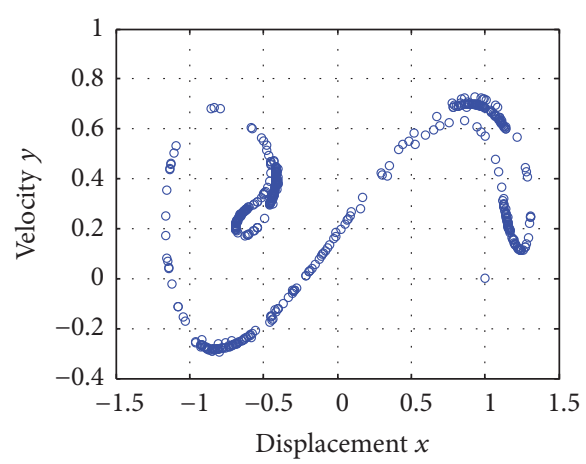

(d)

Figure 5: (a) Top Lyapunov exponents versus time $\left(\omega_{0}=1, \beta=1\right.$, $D=0.4, \gamma=0.1, \omega=1, F=0.4)$. (b) Time history of nonlinear system $\left(\omega_{0}=1, \beta=1, D=0.4, \gamma=0.1, \omega=1, F=0.4\right)$. (c) Phase portrait of nonlinear system $\left(\omega_{0}=1, \beta=1, D=0.4, \gamma=0.1, \omega=1\right.$, $F=0.4)$. (d) Poincare map of nonlinear system $\left(\omega_{0}=1, \beta=1\right.$, $D=0.4, \gamma=0.1, \omega=1, F=0.4)$. 
computations shown in Figures 6(a)-6(d). In Figures 6(a)6(d), we plot the top Lyapunov exponents, the time history, the phase portrait, and Poincare map of nonlinear system. As can be seen from Figure 6(a), the top Lyapunov exponent is computed as $\lambda_{1}=0.044337$ which is positive; the chaotic motion of the system will occur as shown in Figure 6(d). Now if the nonlinear damping effect is considered, namely, $\gamma=0.1$, the other parameters remain the same; the top Lyapunov exponents, the time history, the phase portrait, and Poincare map of nonlinear system are presented in Figures $7(\mathrm{a})-7(\mathrm{~d})$. As can be seen from Figure 7(a), the top Lyapunov exponent turns out to be $\lambda_{1}=-0.01584$, which is negative, and the periodic motion of the system can be seen as depicted in Figure 7(d).

Finally we choose the forcing amplitude as $F=1.0$ which is larger than the critical values of forcing amplitude $\left(F_{c}\right)$ based on the numerical results shown in Figure 2(b). Then the top Lyapunov exponents, the time history, the phase portrait, and Poincare map of nonlinear system are depicted in Figures 8(a)-8(d). The top Lyapunov exponent is estimated as $\lambda_{1}=0.19182$ shown in Figure $8(\mathrm{a})$, which is positive, and the chaotic motion of the system will occur as shown in Figure 8(d).

Based on all the numerical results mentioned above, it is understood that the linear and nonlinear damping can have significant effects on the chaotic behavior of the nonlinear system. That is, the linear and nonlinear damping can influence the nonlinear system into or out of a chaotic regime.

\section{Conclusions}

In the present study, the chaotic behavior of a forced Duffing oscillator subjected to linear and nonlinear damping is investigated by using Melnikov technique. In particular, the critical value of the forcing amplitude of the nonlinear system is calculated by Melnikov technique. The top Lyapunov exponent of the nonlinear system is evaluated by Wolf's algorithm to determine whether the chaotic phenomenon of the nonlinear system actually occurs. As a result, the critical value of forcing amplitude increases linearly with the linear damping coefficient; however, it varies nonlinearly with respect to the driving frequency. Further, the critical value of forcing amplitude decreases nonlinearly with respect to the nonlinear coefficient. To detect the chaotic phenomena of the nonlinear system, the top Lyapunov exponent, the time history, the phase portrait, and Poincare map of the nonlinear system are presented for various cases. It is concluded that the chaotic motion of the nonlinear system with the linear and nonlinear damping occurs when the forcing amplitude exceeds the critical value, and the linear and nonlinear damping have significant effects on the chaotic behavior of the forced Duffing oscillator.

\section{Competing Interests}

The author declares that there is no conflict of interests regarding the publication of this paper.

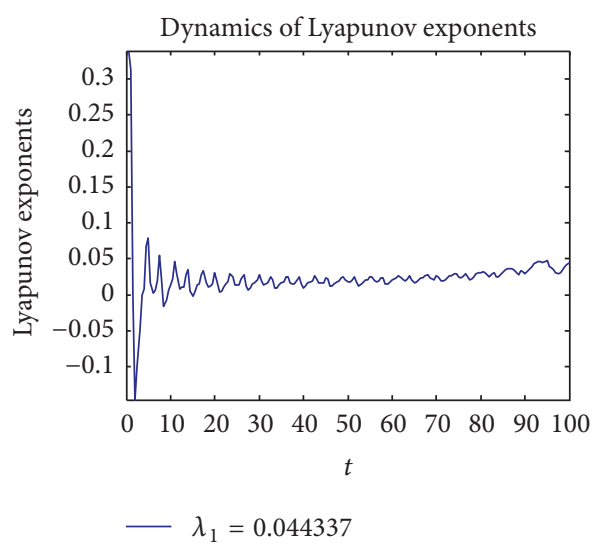

(a)

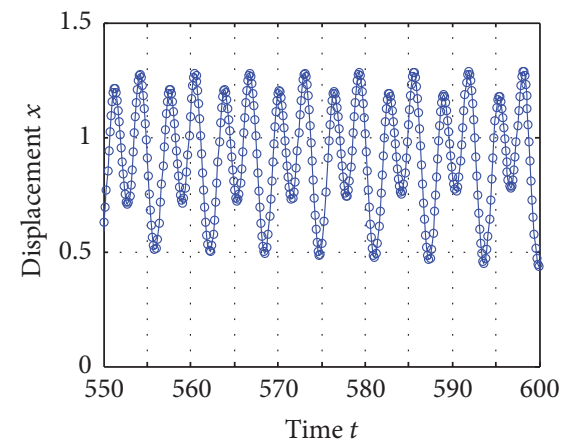

(b)

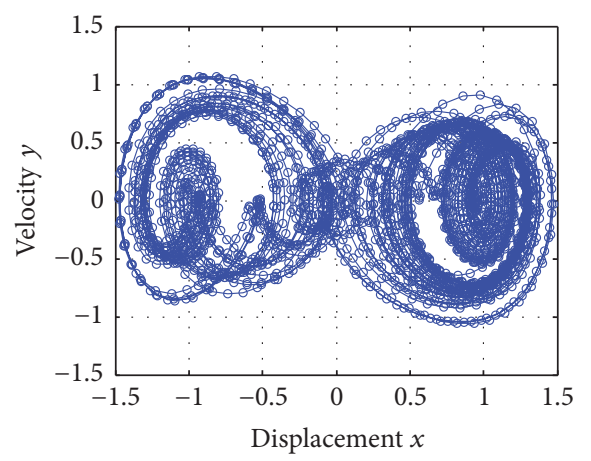

(c)

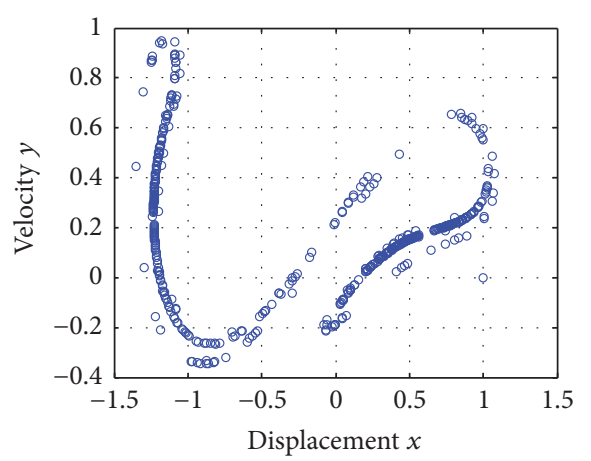

(d)

Figure 6: (a) Top Lyapunov exponents versus time $\left(\omega_{0}=1, \beta=1\right.$, $D=0.4, \gamma=0.0, \omega=2, F=0.8$ ). (b) Time history of nonlinear system $\left(\omega_{0}=1, \beta=1, D=0.4, \gamma=0.0, \omega=2, F=0.8\right)$. (c) Phase portrait of nonlinear system $\left(\omega_{0}=1, \beta=1, D=0.4, \gamma=0.0, \omega=2\right.$, $F=0.8)$. (d) Poincare map of nonlinear system $\left(\omega_{0}=1, \beta=1\right.$, $D=0.4, \gamma=0.0, \omega=2, F=0.8)$. 


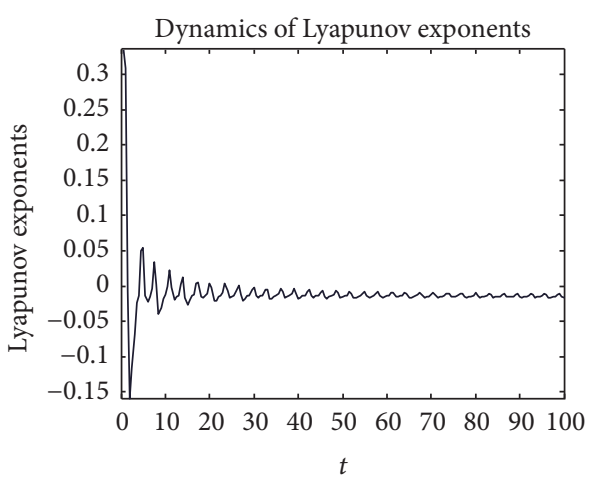

$-\lambda_{1}=-0.01584$

(a)

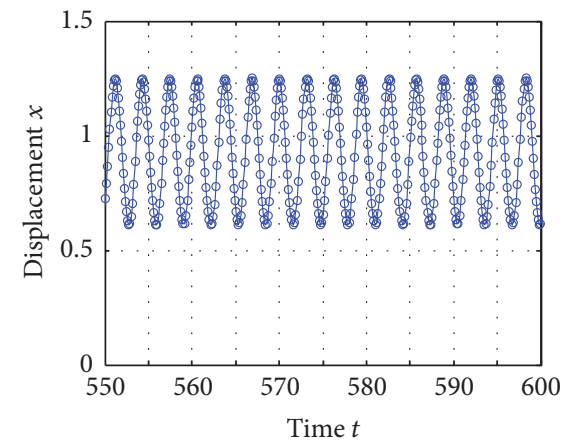

(b)

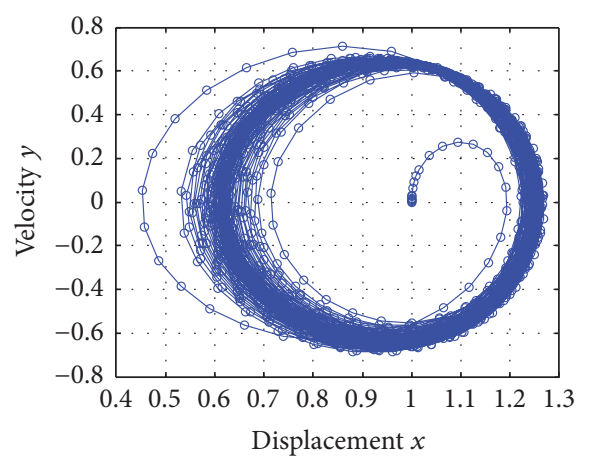

(c)

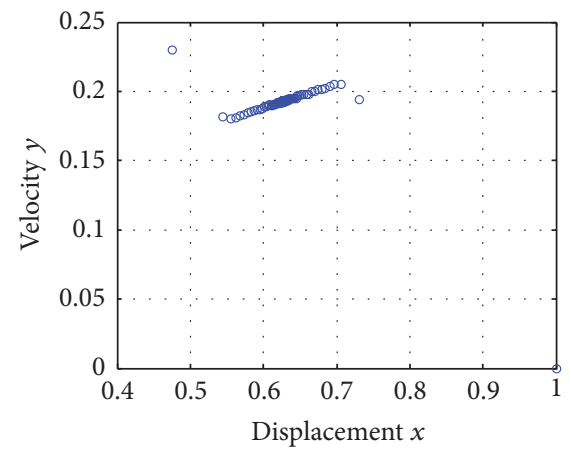

(d)

Figure 7: (a) Top Lyapunov exponents versus time $\left(\omega_{0}=1, \beta=1\right.$, $D=0.4, \gamma=0.1, \omega=2, F=0.8)$. (b) Time history of nonlinear system $\left(\omega_{0}=1, \beta=1, D=0.4, \gamma=0.1, \omega=2, F=0.8\right)$. (c) Phase portrait of nonlinear system $\left(\omega_{0}=1, \beta=1, D=0.4, \gamma=0.1, \omega=2\right.$, $F=0.8)$. (d) Poincare map of nonlinear system $\left(\omega_{0}=1, \beta=1\right.$, $D=0.4, \gamma=0.1, \omega=2, F=0.8)$.

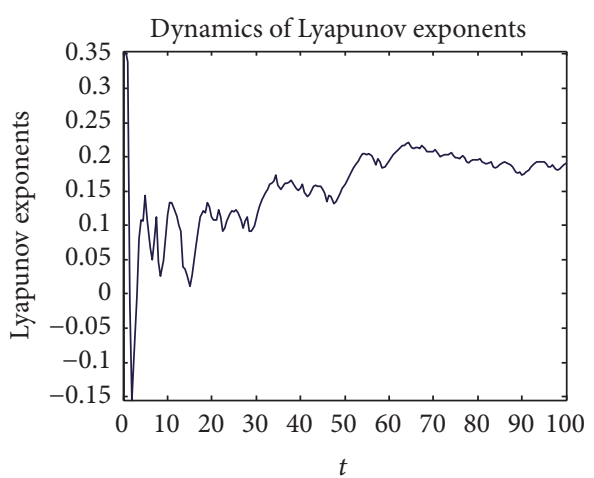

$-\lambda_{1}=0.19182$

(a)

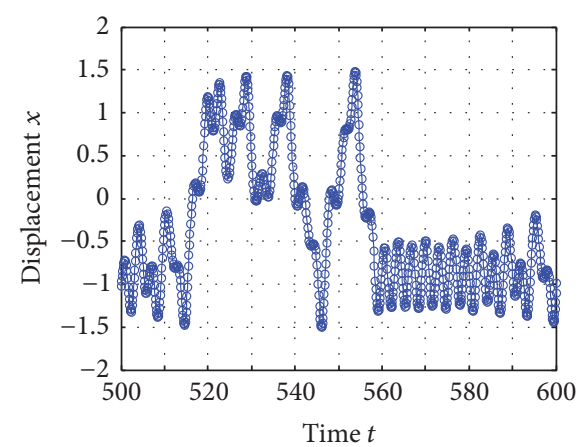

(b)

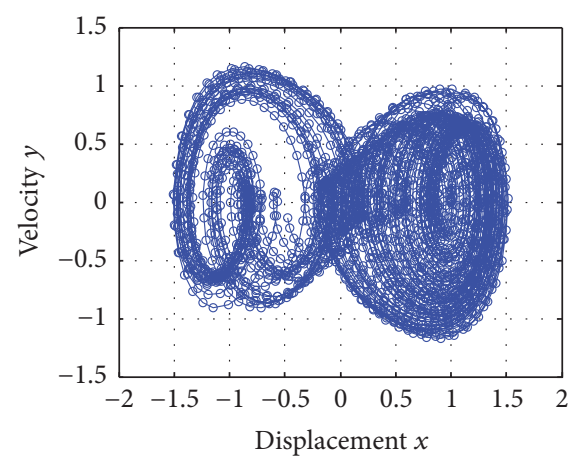

(c)

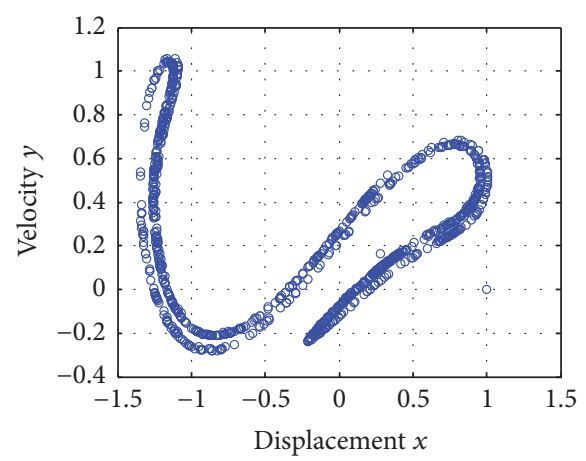

(d)

FIgURE 8: (a) Top Lyapunov exponents versus time $\left(\omega_{0}=1, \beta=1\right.$, $D=0.4, \gamma=0.1, \omega=2, F=1.0)$. (b) Time history of nonlinear system $\left(\omega_{0}=1, \beta=1, D=0.4, \gamma=0.1, \omega=2, F=1.0\right)$. (c) Phase portrait of nonlinear system $\left(\omega_{0}=1, \beta=1, D=0.4, \gamma=0.1, \omega=2\right.$, $F=1.0)$. (d) Poincare map of nonlinear system $\left(\omega_{0}=1, \beta=1\right.$, $D=0.4, \gamma=0.1, \omega=2, F=1.0)$. 


\section{Acknowledgments}

This research was partially supported by the Ministry of Science and Technology in Taiwan through Grant no. MOST103-2221-E-327-011. The author is grateful for the financial support.

\section{References}

[1] Y.-H. Kao and C.-S. Wang, "Analog study of bifurcation structures in a Van der Pol oscillator with a nonlinear restoring force," Physical Review E, vol. 48, no. 4, pp. 2514-2520, 1993.

[2] A. Venkatesan and M. Lakshmanan, "Bifurcation and chaos in the double-well Duffing-van der Pol oscillator: numerical and analytical studies," Physical Review E. Statistical, Nonlinear, and Soft Matter Physics, vol. 56, no. 6, pp. 6321-6330, 1997.

[3] T. Ueda and H. Kawakami, "Unstable saddle-node connecting orbits in the averaged DuffingRayleigh equation," ISCAS, vol. 3, pp. 288-291, 1996.

[4] F. C. Moon, Chaotic and fractal dynamics, A Wiley-Interscience Publication, John Wiley \& Sons, Inc., New York, 1992.

[5] Y.-Z. Wang, F.-M. Li, and Y.-S. Wang, "Influences of active control on elastic wave propagation in a weakly nonlinear phononic crystal with a monoatomic lattice chain," International Journal of Mechanical Sciences, vol. 106, pp. 357-362, 2016.

[6] Y.-Z. Wang and F.-M. Li, "Nonlinear primary resonance of nano beam with axial initial load by nonlocal continuum theory," International Journal of Non-Linear Mechanics, vol. 61, pp. 7479, 2014.

[7] Y.-Z. Wang and F.-M. Li, "Nonlinear free vibration of nanotube with small scale effects embedded in viscous matrix," Mechanics Research Communications, vol. 60, pp. 45-51, 2014.

[8] R. V. Dooren and H. Janssen, "A continuation algorithm for discovering new chaotic motions in forced Duffing systems," Journal of Computational and Applied Mathematics, vol. 66, no. 1-2, pp. 527-541, 1996.

[9] X. Han and Q. Bi, "Bursting oscillations in Duffing's equation with slowly changing external forcing," Communications in Nonlinear Science and Numerical Simulation, vol. 16, no. 10, pp. 4146-4152, 2011.

[10] M. U. Akhmet and M. O. Fen, "Chaotic period-doubling and OGY control for the forced Duffing equation," Communications in Nonlinear Science and Numerical Simulation, vol. 17, no. 4, pp. 1929-1946, 2012.

[11] A. K. Mallik, Principles of Vibrations Control, Affiliated Eastwest Press Private Limited, New Delhi, India, 1990.

[12] B. Ravindra and A. K. Mallik, "Role of nonlinear dissipation in soft Duffing oscillators," Physical Review E, vol. 49, no. 6, pp. 4950-4954, 1994.

[13] M. A. F. Sanjuán, "The effect of nonlinear damping on the universal escape oscillator," International Journal of Bifurcation and Chaos in Applied Sciences and Engineering, vol. 9, no. 4, pp. 735-744, 1999.

[14] J. L. Trueba, J. Rams, and M. A. F. Sanjuan, "Analytical estimates of the effect of nonlinear damping in some nonlinear oscillators," International Journal of Bifurcation and Chaos in Applied Sciences and Engineering, vol. 10, no. 9, pp. 2257-2267, 2000.

[15] J. P. Baltanás, J. L. Trueba, and M. A. F. Sanjuán, "Energy dissipation in a nonlinearly damped Duffing oscillator," Physica D: Nonlinear Phenomena, vol. 159, no. 1-2, pp. 22-34, 2001.
[16] M. Borowiec, G. Litak, and A. Syta, "Vibration of the Duffing oscillator: effect of fractional damping," Shock and Vibration, vol. 14, no. 1, pp. 29-36, 2007.

[17] M. S. Siewe, H. Cao, and M. A. F. Sanjuán, "Effect of nonlinear dissipation on the basin boundaries of a driven two-well Rayleigh-Duffing oscillator," Chaos, Solitons and Fractals, vol. 39, no. 3, pp. 1092-1099, 2009.

[18] G. Litak, M. Borowiec, A. Syta, and K. Szabelski, "Transition to chaos in the self-excited system with a cubic double well potential and parametric forcing," Chaos, Solitons \& amp; Fractals, vol. 40, no. 5, pp. 2414-2429, 2009.

[19] M. S. Siewe, H. Cao, and M. A. F. Sanjuán, "On the occurrence of chaos in a parametrically driven extended Rayleigh oscillator with three-well potential," Chaos, Solitons and Fractals, vol. 41, no. 2, pp. 772-782, 2009.

[20] M. S. Siewe, C. Tchawoua, and P. Woafo, "Melnikov chaos in a periodically driven Rayleigh-Duffing oscillator," Mechanics Research Communications, vol. 37, no. 4, pp. 363-368, 2010.

[21] A. Sharma, V. Patidar, G. Purohit, and K. K. Sud, "Effects on the bifurcation and chaos in forced Duffing oscillator due to nonlinear damping," Communications in Nonlinear Science and Numerical Simulation, vol. 17, no. 6, pp. 2254-2269, 2012.

[22] Y.-B. Liu, Y.-S. Chen, and Q.-J. Cao, "Analysis method of chaos and sub-harmonic resonance of nonlinear system without small parameters," Applied Mathematics and Mechanics, vol. 32, no. 1, pp. 1-10, 2011.

[23] V. K. Melnikov, "On the stability of the center for time periodic perturbations," Transactions of the Moscow Mathematical Society, vol. 12, pp. 1-57, 1963.

[24] J. Guckenheimer and P. Holmes, Nonlinear Oscillations, Dynamical Systems, and Bifurcations of Vector Fields, vol. 42, Springer, New York, NY, USA, 1983.

[25] A. Wolf, J. B. Swift, H. L. Swinney, and J. A. Vastano, "Determining Lyapunov exponents from a time series," Physica D: Nonlinear Phenomena, vol. 16, no. 3, pp. 285-317, 1985.

[26] Y.-Z. Wang and F.-M. Li, "Dynamical properties of Duffingvan der Pol oscillator subject to both external and parametric excitations with time delayed feedback control," Journal of Vibration and Control, vol. 21, no. 2, pp. 371-387, 2015.

[27] K. Yagasaki, "A simple feedback control system: bifurcations of periodic orbits and chaos," Nonlinear Dynamics, vol. 9, no. 4, pp. 391-417, 1996. 


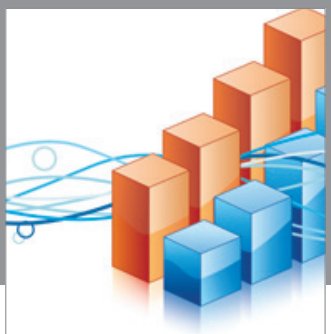

Advances in

Operations Research

vatem alat4

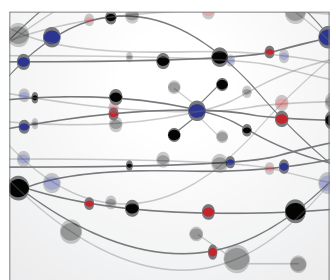

\section{The Scientific} World Journal
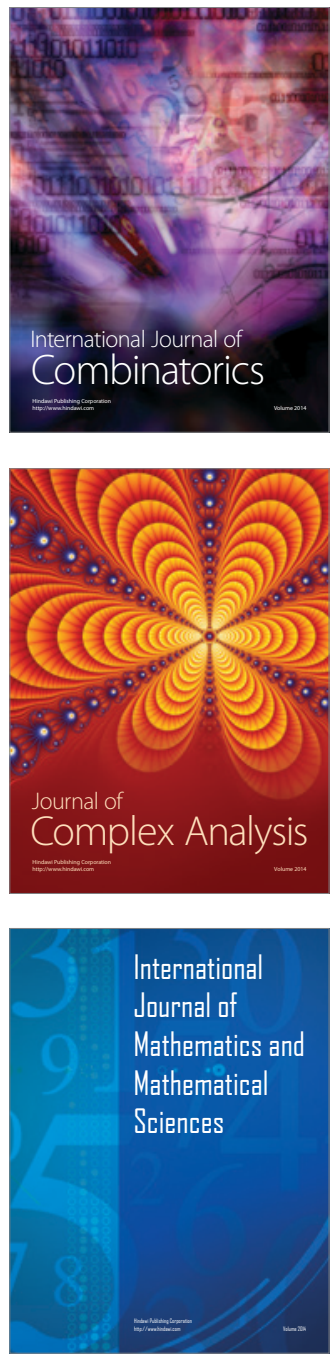
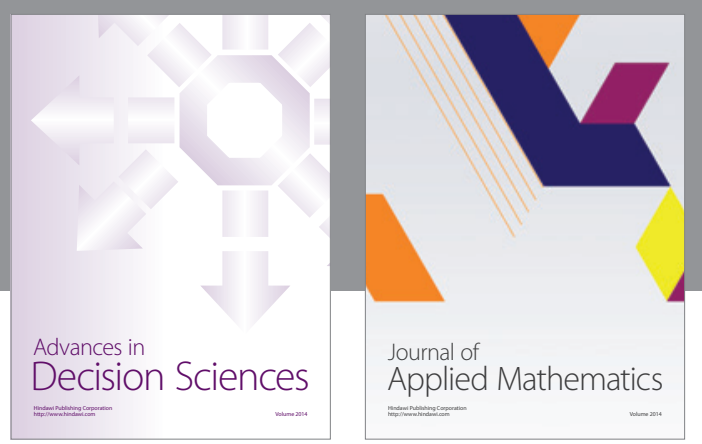

Algebra

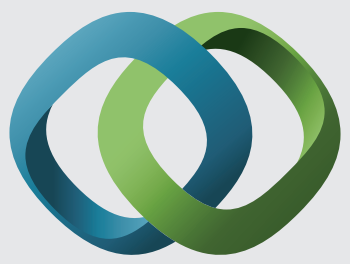

\section{Hindawi}

Submit your manuscripts at

https://www.hindawi.com
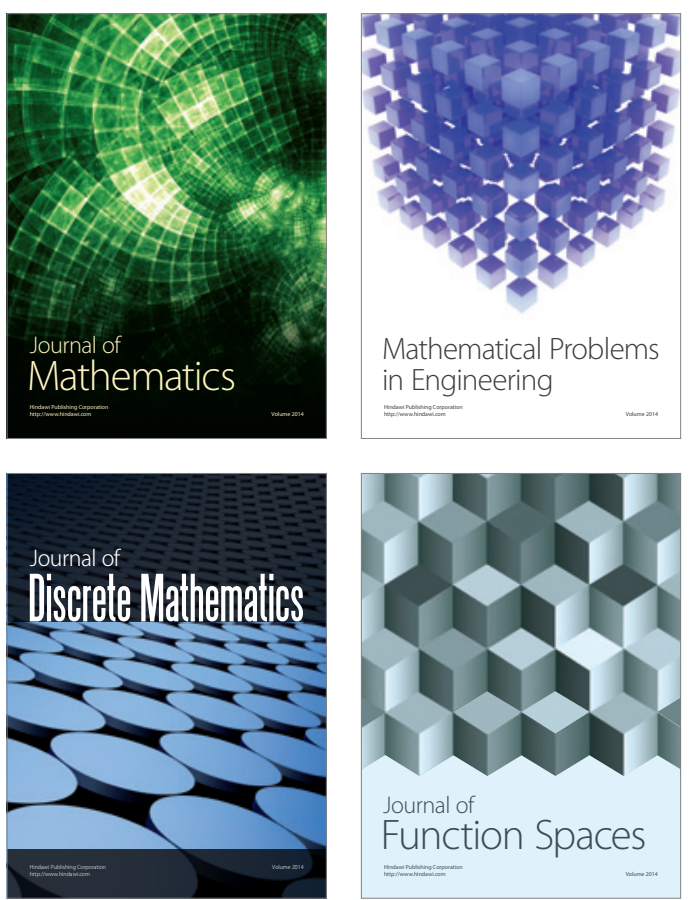

Mathematical Problems in Engineering
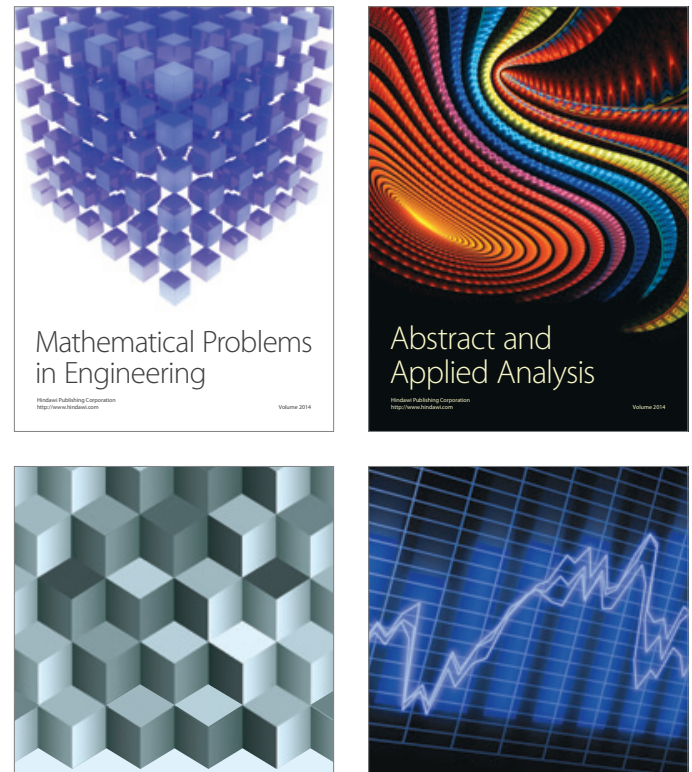

Journal of

Function Spaces

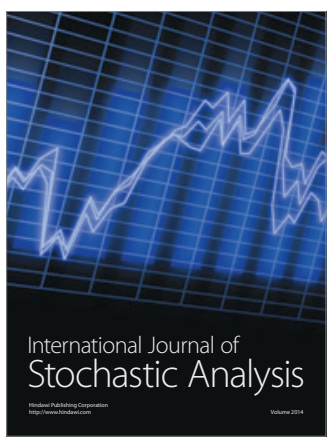

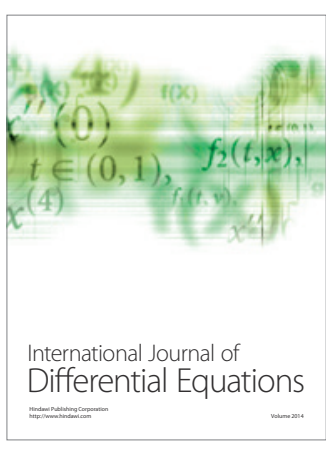
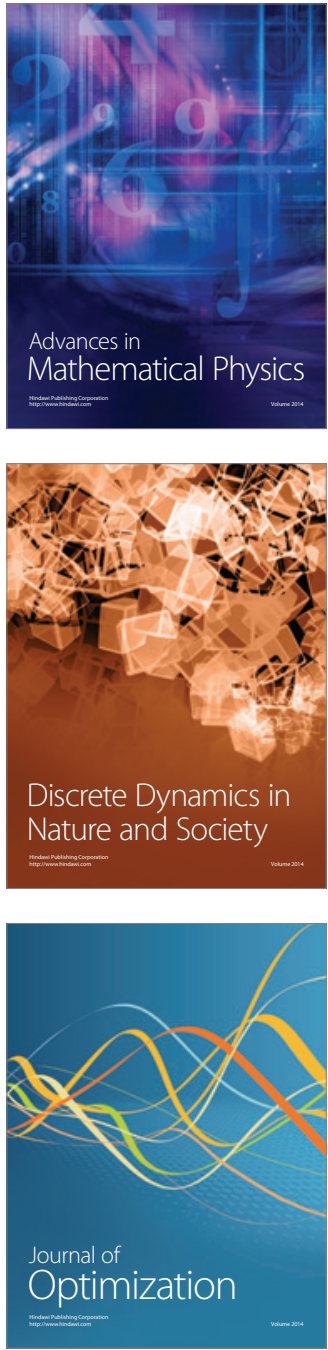